Journal of Engineering and Applied Sciences 14 (18): 6782-6787, 2019

ISSN: 1816-949X

(C) Medwell Journals, 2019

\title{
Design of Mobile Robot for Obstacle Avoidance using Microcontroller
}

\author{
Ayam Mohsen Abbass \\ Department of Computer Engineering, Mustansiriyah University, Baghdad, Iraq \\ ayammohsen@uomustansiriyah.edu.iq, +9647711581851
}

\begin{abstract}
Mobile robot for obstacle avoidance is a simple machine with collision avoidance. In addition, it has very big to exploration in future development. By using this simple collision avoidance system, a lot of diversity and new mobile robot which have different functions is invented. The avoidance of obstacle in real time is consider as a one of key issues to success the mobile robot application systems. In this study mobile robot with a voiding the collision is developed by using onboard sensors and the microcontroller. The designed robot is capable of moving in an environment which has obstacles with avoiding the collisions. It is a robot platform which has a four wheels and an ultrasonic range sensor that is fixed in the front of it for scanning the environment. The ultrasonic range sensor will feedback a triggered signal to the microcontroller wirelessly using Bluetooth that programmed in Arduino IDE.
\end{abstract}

Key words: Mobile robot, Arduino, visual basic, obstacle avoidance, ultrasonic range sensor, developed

\section{INTRODUCTION}

Exploration in robot field means the capability to search and work in the surrounding without obstacle collides, the capability to conclude the current position and the capability to arrive at desired goal locations. So, in exploration system may include the following: the system that find the robot positioning, map construction and path planning (Zaki et al., 2014). The mobile robot behavior is accomplished by the cooperation between software program (task), hard ware components of the mobile robot (sensors, motors) and the surrounding (Ismail et al., 2016). The ability to move means how the robots can moved freely by themselves, so that, they can achieved their tasks. In the case that the mobile robot is guided by the controller which is controlled by the human remotely it is called as non-autonomous and these kinds of robots is expected for interpreting the instructions that given by the human controllers (Mutlu and Uyar, 2012).

In autonomous mobile robot with sensing ability for avoiding an obstacle there is a lot of researches and different types of sensors are usefully used in mobile robot. By Mutlu and Uyar (2012) show some introductory results of discover the movable obstacles like as human moves by fixing a camera on the mobile robot the detection method for moving objects is good but in some cases is failed due to many factors like object distance color of object and the reflection of light. By Kim and Do (2012) presents a development and implementation of the systems in a mobile robots with obstacle avoidance in true time and these systems are controlled by neural network and employ ultrasonic range sensors with difficult techniques of made decision in progress. By Medina-Santiago et al. (2014) navigation system is combined the acknowledgment and dead reckoning is used to give satisfied operation, designed a microcontroller system to control the mobile robot navigation with avoiding obstacles. By Rejab and Naji (2011) present that to accomplish a system with obstacle avoidance in true time for wheeled mobile robots, three ultrasonic range sensors are used in order to enable the robot frequently detects the environment, obstacle avoidance and changes place toward the required position and to reaches the target. By Ahmed (2018) remotely controlled the mobile robot by using android phone with sensors such as ultrasonic sensor, IR sensor and motion sensor, the designed robot is able to navigate to the places of flowerpots in the area and distribute water.

\section{MATERIALS AND METHODS}

This study of the research explains the block diagram of the designed system and the hardware system design with describes the required components.

System block diagram: In this research, the software and hardware components are combined together to produce reliable system. The Arduino Uno will be used as the 


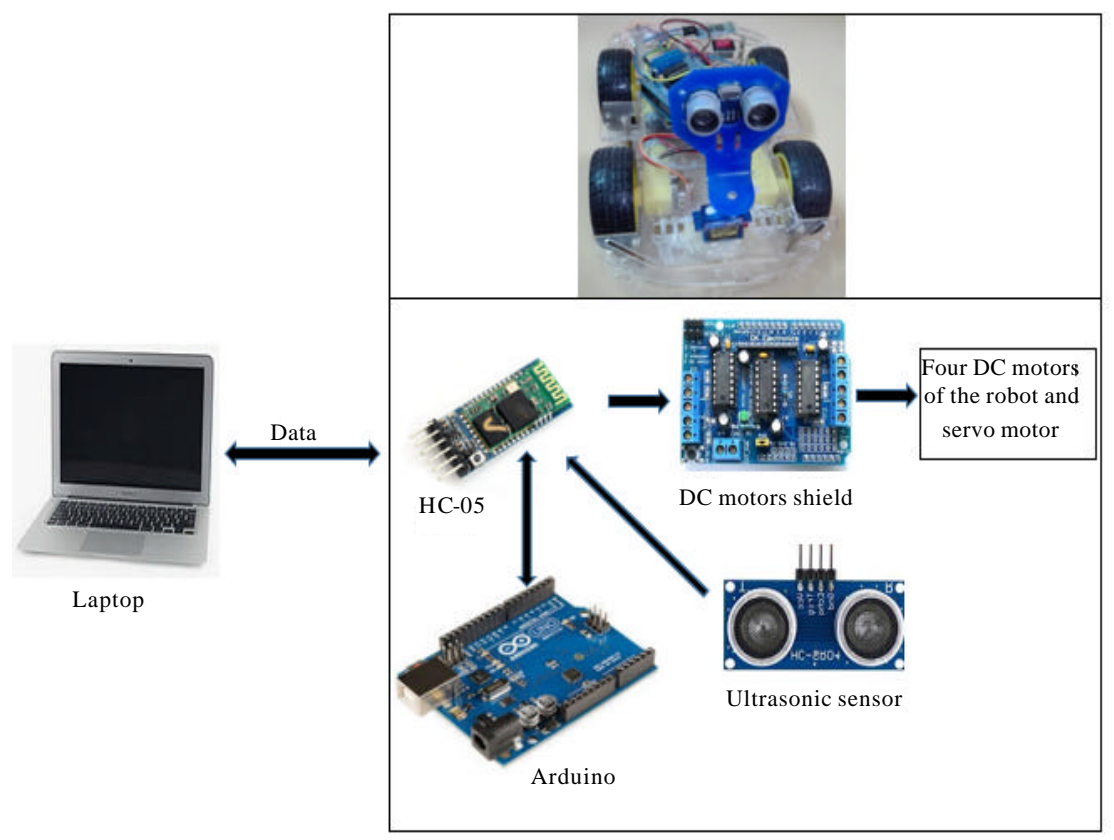

Fig. 1: The mobile system designed

interfacing for the robot and it is controlled by using wireless communication technique (Bluetooth). The designed system overview is shown in Fig. 1.

Hardware system design: Mobile robot design was accomplished to be modified easily and adapted to new and future research. The hardware components which are used to design the desired mobile robot are the microcontroller, DC motor for car prototype, DC motor shield, Bluetooth module, ultrasonic range sensor, servo motor and DC battery. The microcontroller used is an Arduino Uno as the brain of the mobile robot to control the mobile robot connected components (motors and sensors).

Moving control system of mobile robot: The microcontroller sends the control signals to the two of the four DC motors at a time by using the motor driver shield because Arduino is not able to control DC motors by its own because the current that is generating is very low and is not able to reverse, so, the direction of the motor will not change. To find solution for this used a motor driver to help the Arduino to control the DC motors. The block diagram of mobile robot moving control system is shown in Fig. 2.

The DC motor shield is a driver shield used to drive multi DC motors, Fig. 3 shows a motor shield. This driverhas two of L293D driver for DC motors, the voltage ranges between (4.5VDC-16 VDC) it can drive four

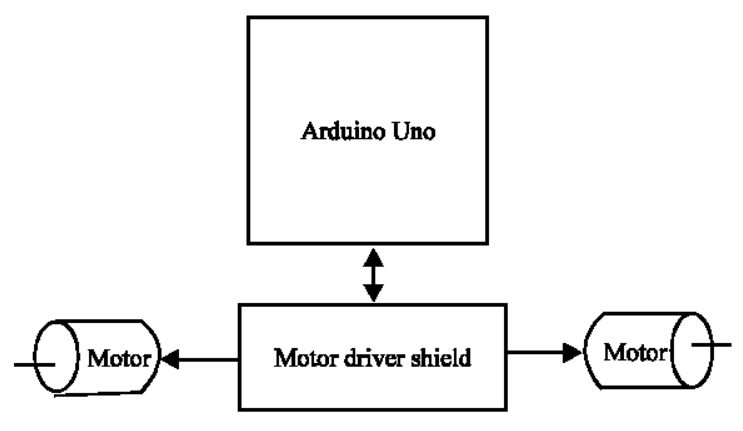

Fig. 2: Block diagram of the robot moving control system

bi-directional motors with eight bit speed of individual with selection speed (256 speeds) and it provides separation of logic and power connections for each motor. The motor for car prototype is a gear DC motor, the range of Voltage is (3-6 V) that works with it this motor rotates a tire which is connected with it. It has a single axis and the Rotation Per Minutes (RPM) equal $125 \mathrm{R} / \mathrm{min}$ used a gear box for tracing a robot. Figure 4 shows a DC gear motor used for the robot prototype.

Obstacle avoiding system of mobile robot: A block diagram of an obstacle avoidance system is shown in Fig. 5. The servo motor turns the ultrasonic sensor to three directions to read data continuously and send them to the Arduino when an obstacle is detected the Arduino 


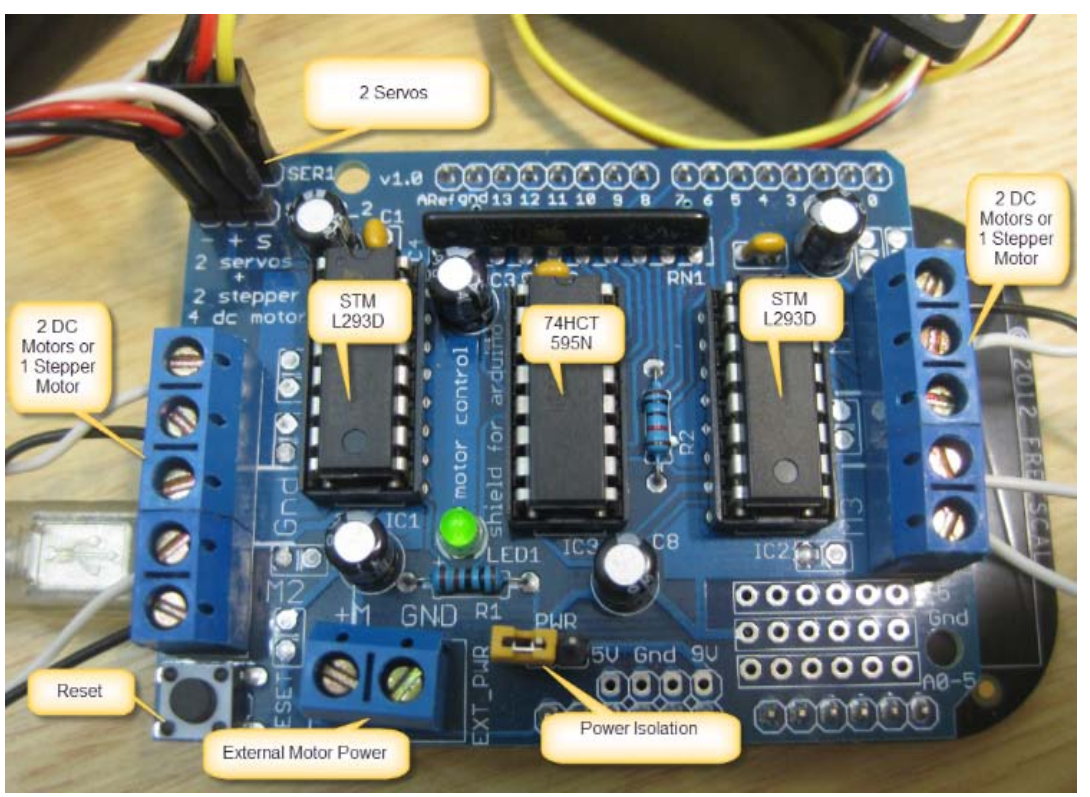

Fig. 3: Multi-motor driver shield (2-L293D)

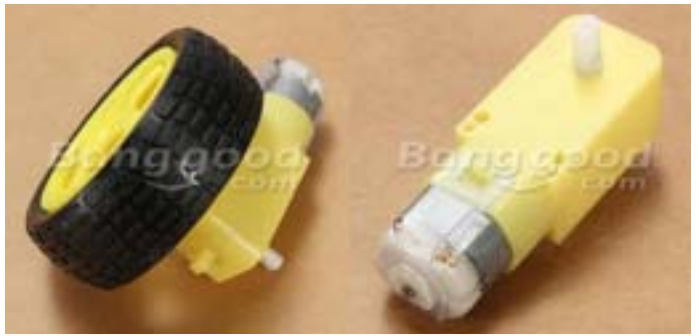

Fig. 4: DC gear motor

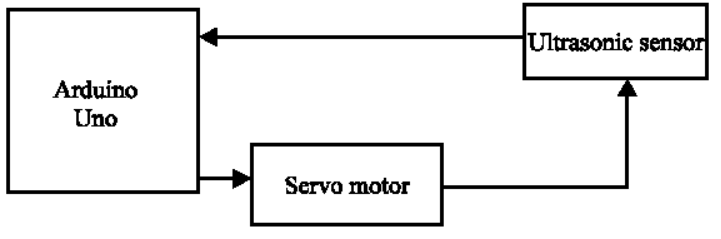

Fig. 5: Block diagram of the robot obstacle avoiding system

sends signal to the DC motors to decide which side to turn the robot to avoiding any collision to the obstacles. A servomotor is a linear or rotary actuator that permits controlling the linear or angular position precisely acceleration and velocity. It contains a motor that coupled with sensor for sensing the position feedback. It also needed a relatively complicated controller. This type of motor is suitable to use in controlling a closed-loop system. Servomotor is shown in Fig. 6.

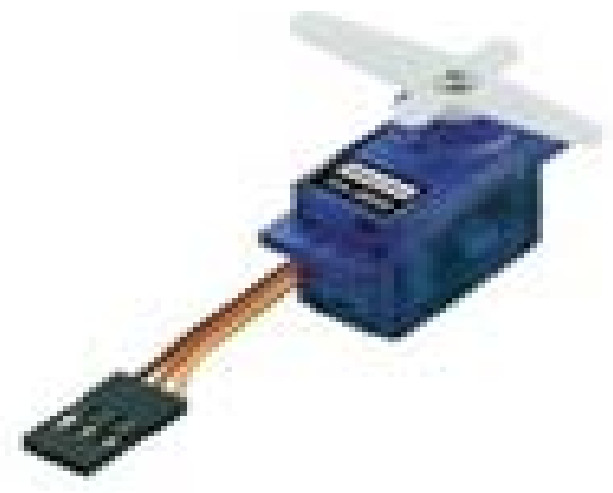

Fig. 6: Servomotor

The ultrasonic transducer is convert an electrical signals to ultrasound waves and vice versa. It is also named an ultrasound transceiver besides it is ultrasound sensor is indeed transceiver because it can be both of sensing and transmitting signals. This device based on a principle like transducer which is applied in a sonar systems and the radar which evaluates the target attributes by interpreting an echo from sound or radio waves. Ultrasonic sensor generates sound waves with high frequency and evaluates the received back echo by a sensor then measures the interval of a time between the sending of signal and the receiving of echo to find the object distance $(3-5 \mathrm{~m})$. The circuit diagram for connecting the ultrasonic sensor to the brain (Arduino) 


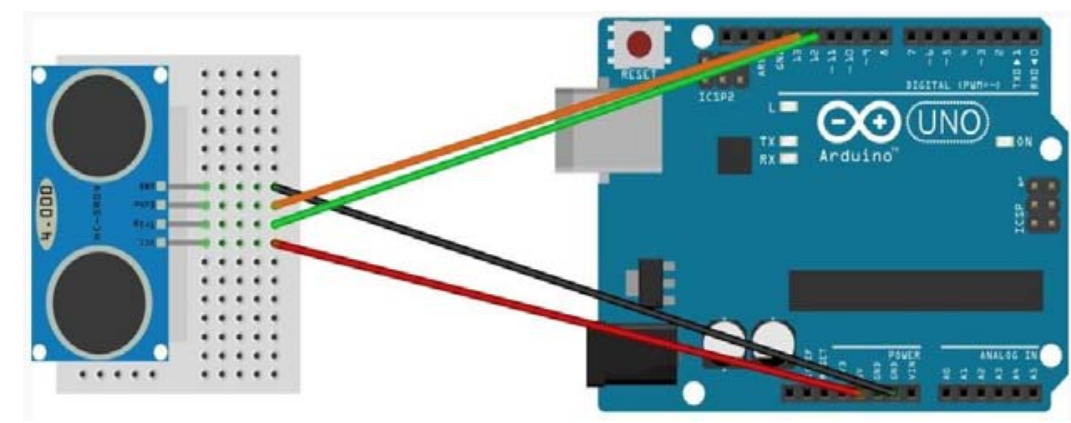

Fig. 7: Circuit diagram for connecting ultrasonic sensor with Arduino

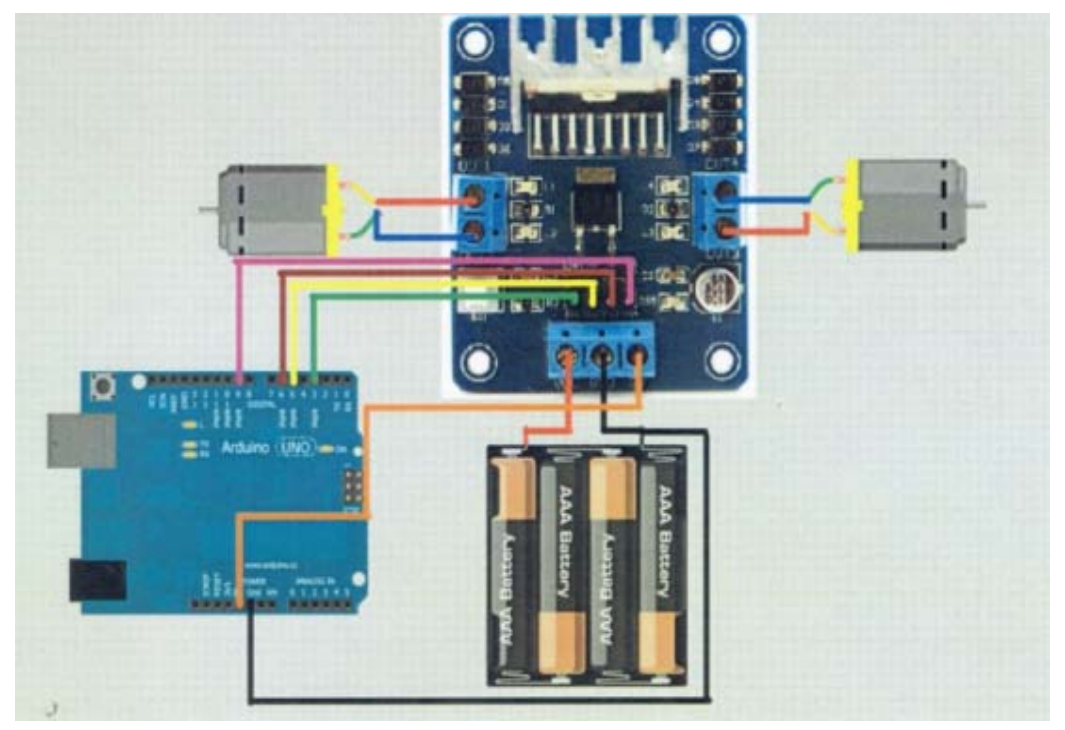

Fig. 8: The circuit diagram for driving two motors

is shown in Fig. 7 in this circuit PIN 12 and 13 in an Arduino are used to send and receive the sound waves with in trigger and echo pins of ultrasonic sensor, respectively. The VCC of ultrasonic is connected with the Arduino VCC and the GND of the ultrasonic is connected with the Arduino GND.

Explanation of linkage: In this research the DC motors of the mobile robot are connected to the motor shield where the front DC motors that lie on the right and left hand sides are connected to the PIN M4 and M2 of the motor shield, respectively. The back DC motors that lie on the right and left hand sides are connected to the PIN M3 and M1 of the motor shield, respectively. The circuit diagram for driving two motors is shown in Fig. 8.

Bluetooth is a wireless communication which is utilized for transmitting voice and data through short distance with radio waves in high speed. Easily, HC-05 is interfaced with Arduino (Uno) as shown in Fig. 9. The Bluetooth
VCC is connected to Arduino's VCC and the Bluetooth GND is also connected to the Arduino GND. The RX for Bluetooth is connected to the PIN 1 in the Arduino and the Bluetooth TX is connected to the Arduino PIN 0. The servo motor is connected to the servo pins which are located in the motor shield. The lithium battery is connected with the GND and M+ of the motor shield, so that, the robot is supplied with energy that helps it to move and execute the instructions.

Explanation of software interface: In this research mobile robot is controlled by using two approaches in the first using keyboard for controlling the movement of the robot and in the second use an interface programmed in visual basic for controlling the movement of the robot. The servo angle was defined at the beginning of the robot's work and then the Bluetooth code was defined. After that the pin of the ultrasonic was defined with output mode. 


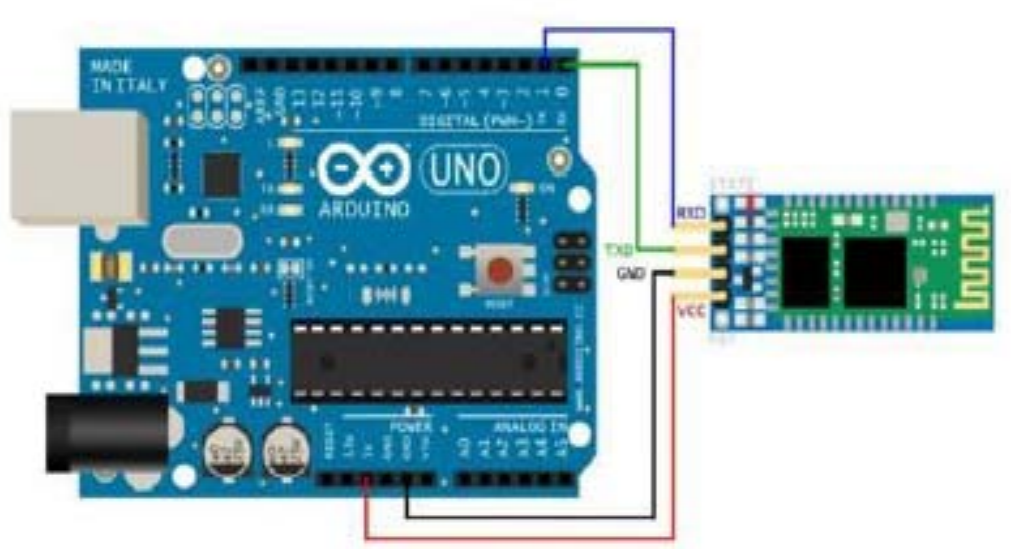

Fig. 9: Interface $\mathrm{HC}-05$ with Arduino

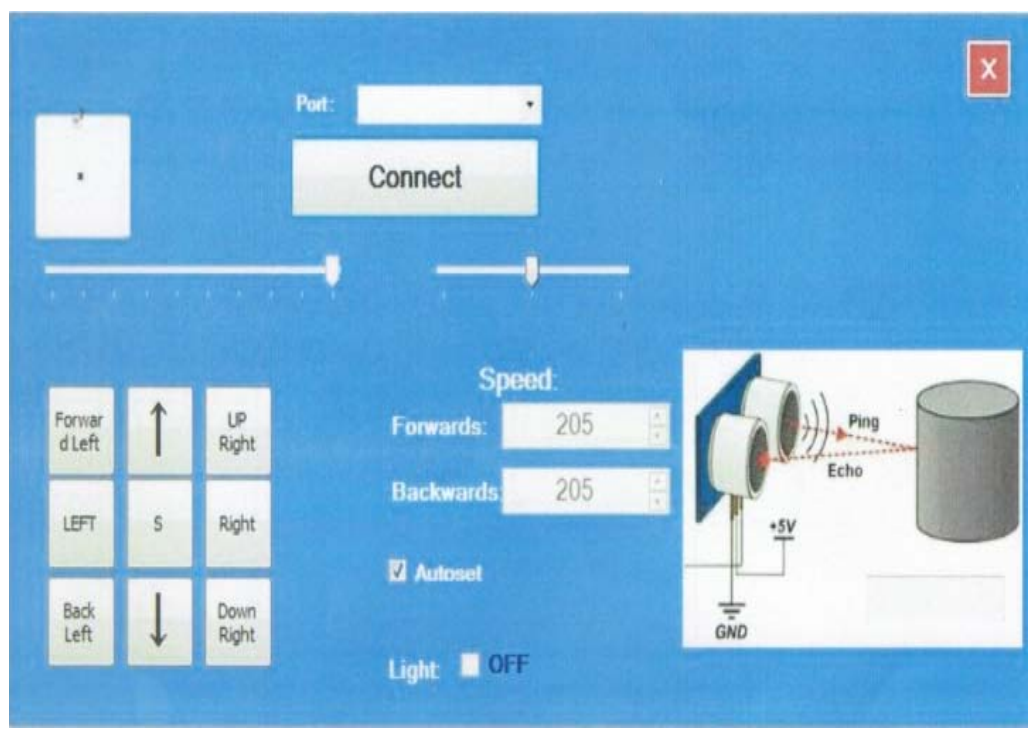

Fig. 10: The visual basic interface

Then determine the measurement distance at this the ultrasonic sense an obstacle to avoid collision. It checks the distance if it is $<50 \mathrm{~cm}$, the servo will move at 180 degrees and calculates the distance if it is $<50 \mathrm{~cm}$, forward move the right side wheels and backward move the left side wheels. This movement will result in the rotation of the robot to the left hand side.

Decide the direction of robot movement according to the switch instruction options if option is (A) all wheels will be moved forward while in the case of option (B) all the wheels will be moved backward. According to the switch instruction option, direct moving the robot to the left or right, if option is $(\mathrm{R})$ caused to move all the wheels forward but by programmed the movement of the wheels in the right hand side is faster than the movement of the wheels in the left hand side which will lead to the change of direction of the robot to the right hand side. But when it is (L) caused all the wheels move forward but by programmed the movement of the wheels in the left hand is faster than the movement of the wheels in the right hand side which will lead to the change of direction of the robot to the left side. The principle of moving one of the robot sides at a certain speed and move to the opposite side in the same direction but quickly less than will lead to rotate of the robot to direction where the movement of the wheels faster.

Figure 10 shows the Graphically User Interface (GUI) window in visual basic designed that used for several tasks such as to interface the mobile robot with the laptop to achieve wireless communication through Bluetooth technique for controlling the ultrasonic sensor by limit the distance permission for sensing an obstacle from 


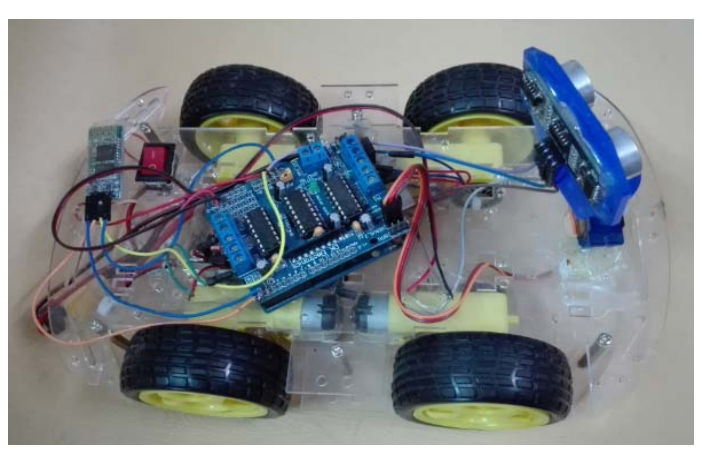

Fig. 11: Mobile robot interfacing

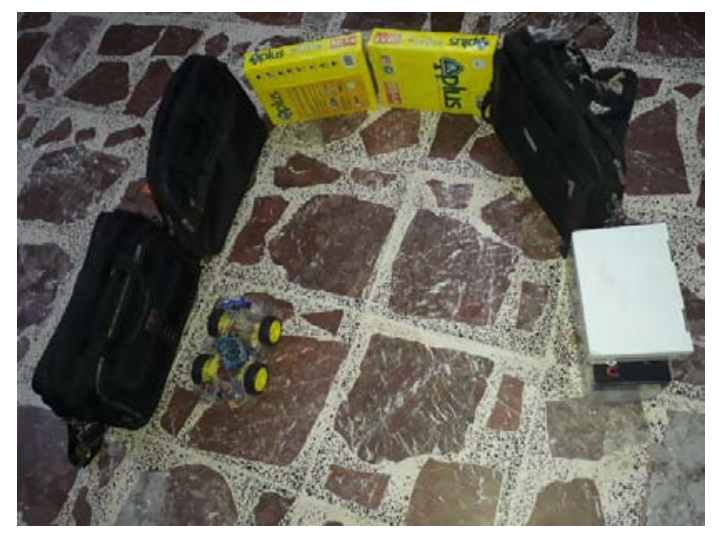

Fig. 12: An example of movement of mobile robot

the robot to change its direction and for controlling the speed of servo motor which is connected with ultrasonic sensor. Figure 11 shows an interface of the mobile robot, microcontroller (Arduino), motors shield, ultrasonic range sensor and HC-05 module. Figure 12 shows an example for moving the mobile robot with obstacle avoidance.

\section{CONCLUSION}

The primary requirement for independent mobile robot is obstacle avoidance. The robot collects the information about the surrounding places by using sensors fixed on the mobile robot. In this research a low cost method is achieved for an obstacle avoidance of a mobile robot by using an ultrasonic sensor just one. A mobile stand-alone obstacle avoiding robot is designed using an ultrasonic sensor to detect the obstacles and then make a decision using Arduino based microcontroller to avoid the obstacle and move the robot to another direction free of obstacles. In this research two ways are used for controlling the mobile robot for avoiding an obstacle, the first is achieved by using keyboard and the second is achieved by using GUI in visual basic program to provide an alternate way for controlling the robot with the same hardware required.

\section{ACKNOWLEDGEMENT}

The researcher would like to thank AL-Mustansiriyah University (www.uomustansiriyah.edu.iq) Baghdad, Iraq for it is support in the present research.

\section{REFERENCES}

Ahmad, B., 2018. Outdoor mobile robotic assistant micro-controller module (Arduino), firmware and infrared sensor circuit design and implementation, operating principle and usage of pirmotion sensor. Intl. J. Artif. Intell. Appl., 9: 1-8.

Ismail, R., Z. Omar and S. Suaibun, 2016. Obstacle-avoiding robot with IR and PIR motion sensors. IOP. Conf. Ser. Mater. Sci. Eng., 153: 1-6.

Kim, J. and Y. Do, 2012. Moving obstacle avoidance of a mobile robot using a single camera. Procedia Eng., 41: 911-916.

Medina-Santiago, A., J.L. Camas-Anzueto, J.A. Vazquez-Feijoo, H.R. Hernandez-de Leon and R. Mota-Grajales, 2014. Neural control system in obstacle avoidance in mobile robots using ultrasonic sensors. J. Appl. Res. Technol., 12: 104-110.

Mutlu, L. and E. Uyar, 2012. Control and navigation of an autonomous mobile robot with dynamic obstacle detection and adaptive path finding algorithm. IFAC. Proc., 45: 355-360.

Rejab, K.H. and S.K. Naji, 2017. Design and implementation of intelligent mobile robot based on microcontroller by using three ultrasonic sensors. Intl. J. Curr. Eng. Technol., 7: 1-6.

Zaki, A.M., O. Arafa and S.I. Amer, 2014. Microcontroller-based mobile robot positioning and obstacle avoidance. J. Electr. Syst. Inf. Technol., 1: 58-71. 